\title{
EVALUATION OF QUALITY OF LIFE ISSUES AFTER DIFFERENT MANAGEMENT POLICIES FOR VESTIBULAR SCHWANNOMAS: WHAT ARE WE MEASURING?
}

\author{
Badr Eldin Mostafa
}

\author{
Department of Otorhinolaryngology, Head and Neck Surgery, Faculty of Medicine, \\ Ain-Shams University, Cairo, Egypt
}

\begin{abstract}
PURPOSE: To compare the different quality of life measures used to report the outcomes of the different forms of management of vestibular schwannomas.

METHODS: Literature search and critical evaluation of outcome measures and comparison between different studies.

RESULTS: A total number of 6749 patients were included in 31 studies. Eighteen studies used the SF36 forms, 17 - custom questionnaires, 7 - the GBI and 8 used various other questionnaires. The most commonly reported handicaps included hearing loss, facial nerve paralysis, tinnitus, gait and balance disorders, pain and headache, psychological and cognitive problems, loss of taste and lacrimation and facial hypoesthesia.

CONCLUSIONS: When evaluating QOL issues in patients with vestibular schwannoma, the type of intervention as well as the pre-interventional status should be considered. More specific comprehensive systems of evaluation should be devised through the cooperation of multiple centers with different management policies and variable patient populations as well.
\end{abstract}

Keywords: vestibular schwannoma, quality of life measures, microsurgery, follow-up

\section{INTRODUCTION}

Vestibular schwannomas are benign tumours with minimal early symptoms, but considerable morbidity can result during their natural progression or subsequent to treatment. Consequently, QOL issues become of paramount importance when considering treatment options. The current methods of man-

Address for correspondence:

Prof. Badr Eldin Mostafa

Professor of ENT-HNS

Faculty of Medicine, Ain-Shams University,

34 a El Higaz Str.

11351 Heliopolis, Cairo, Egypt

e-mail:bemostafa@med.asu.edu.eg

Received: November 4, 2015

Accepted: December 1, 2015 agement have entirely different goals. Surgery aims at completely removing tumors, radiosurgery aims to control tumor growth whereas radiologic surveillance aims to manage patients by serially measuring tumor size on the assumption that a significant proportion of vestibular schwannomas do not grow $(1,2)$. The perception of treatment outcomes also differ between the surgeons and their patients. The impact of different treatment modalities on the patient's QOL using the general impairment, disability and handicap scales may not reflect the specifics of the disease and the consequences of its management (3, 4). Disease-related measures should be designed to assess the true impact of treatment options and assist in a comprehensive discussion with patients, preplanning of rehabilitative processes and long-term support of the patients regarding vocational and social issues. The aim of this work is to review the re- 
cent literature on the effects of management on various quality of life indices and attempt to sort out the most relevant points, which need to be included in any scheme to help in the decision making and counseling of patients.

\section{MATERIAL AND METHODS}

A search was performed on PubMed with the following search criteria:

* vestibular schwannoma/acoustic neuroma;

* microsurgery;

* gamma knife/radiation;

* conservation/wait and rescan/wait and see;

* follow-up;

* quality of life measures;

* in humans and in English language.

Thirty-one representative studies were included (1996-2010). All articles described the effect of one or more management policies on the patients' quality of life. Each article was analyzed for the type of management, the method of assessment and the main conclusion as regards the highest impact of the management policy on the patients' quality of life. The results were compared and a list of the most important causes of impairment made.

\section{RESULTS}

A total number of 6749 patients were included, 5252 underwent surgery, 874 -radiosurgery and 470 were scheduled for a wait and see policy. Of these studies, 18 used the SF36 forms, 17 - custom questionnaires, 7 - the GBI and 8 used various other questionnaires. Sixteen studies used more than one evaluation form. In all studies, age, gender, tumor size or audiometric data did not lead to any statistical differences in the result. Women tended to have lower scores, but this finding is present in normative data.

The most commonly reported handicaps included hearing loss, facial nerve paralysis, tinnitus, gait and balance disorders, pain and headache, psychological and cognitive problems, loss of taste and lacrimation and facial hypoesthesia.

\section{Custom questionnaires:}

Hearing loss is one of the major effects of therapy (5). However hearing impairment was not a major factor that impacted patients' QOL after surgery; but paradoxically, hearing preservation did not lead to an improvement in the reported measures (6). It is assumed that patients had already experienced some degree of hearing loss prior to surgery and most were acquainted with a changed lifestyle.

The second commonest handicap was facial nerve dysfunction including taste and salivation. The impact of facial paralysis and sensory impairments varies between studies from insignificant to extremely troublesome (7-9). Facial nerve impairment and physical pain were the two major symptom factors that had a significant impact on QOL of patients after surgery. These two symptoms together might result in weakened psychological adjustment to illness in the area of social, vocational, and domestic environment, as well as patients' sexual relations (4).

Loss of balance and pain variably affected patients' quality of life. In some studies, the effect was not significant whereas in others they are usually associated with a significant negative impact on social functioning (10). Hearing and balance domains reflect not just symptom scores but also functional and societal limitations and so it made sense that these domains correlated most strongly with the SF-36 physical role limitation domain (11).

Psychosocial morbidity such as depression, anxiety and loss of confidence may be present in up to a quarter of surveyed patients. Fatigue appeared to be associated with other symptoms such as hearing impairment, balance disturbance and facial paralysis. For example, listening, walking and/or enunciation had become more difficult and therefore required higher levels of concentration. Hearing loss was a more difficult symptom than facial paralysis because it interfered with communication. Anxiety was also related to hearing problems. $(7,12,13)$. The participants who reported higher levels of psychological wellbeing were those who experienced less severe physical symptoms and believed that they had received a good treatment outcome. Uncertainty about prognosis was more prevalent among radiation patients (14).

\section{General questionnaires:}

In the articles using SF36 or SF12 forms, the most frequently reported handicaps were a diminution in physical, role physical and mental items. Hearing loss was the most distressing handicap followed by facial paralysis. Paradoxically, hearing pres- 
ervation did not improve SF36 scores and facial dysfunction did not lower the scores. There was no significant difference in terms of Glasgow Benefit Inventory scores between patients with and without facial dysfunction, nor between those with different House-Brackmann grades (15). Lower scores in social functioning were due to balance problems not hearing loss. A study on 199 patients using well-recognized questionnaires suggested that vertigo is the symptom which causes the most pronounced negative effect on scores for quality of life with unilateral hearing loss and tinnitus having less effect (16). Increased numbers of postoperative symptoms and/or larger tumors are associated only with worse physical functioning. More bodily pain results in a more negative perception of general health as well as continuous medical consultation (15-18).

In addition, the perception of morbidity varies with age and gender. In order of frequency, men reported hearing loss, balance problems, perioperative surgical experience, and eye and facial weakness, and women reported hearing loss, facial weakness, eye problems, and headache. Facial weakness was a morbidity more often reported for men and women who had large tumors, who were young, or who had undergone the retrosigmoid approach. Balance dysfunction was significant in patients older than 75 years of age. In patients with small tumors, headaches and balance problems were frequently reported (19). The distress experienced by patients with facial paralysis following vestibular schwannoma surgery does not correlate with the grade of the paralysis as attributed by surgeons. Patients with minimal disturbance of facial nerve function may experience great personal distress $(7,13)$.

There were more impairments $51 \%$ (hearing loss, balance) than disabilities 34\% (difficulty in sound localization, eating problems) and more disabilities than handicaps 15\% (reluctance to attend large gatherings, loss of work) (12).

This ability to resume daily activities was independent of the occurrence of hearing problems, in contrast to ataxia, dysgeusia and headache and facial paresis SF-36 has demonstrated, however, that only headache, and not age, sex, hearing or facial function after surgery, was associated with lower scores in any category (physical role limitations and bodily pain).
Headache and facial problems were furthermore the main reason for continuous medical consultation in about one-third of patients after surgery (18-20).

In most reviewed studies the QOL of patients undergoing radiotherapy was better than any form of surgery; patients in the wait and see groups had obviously no change in their quality of life post-diagnosis. One study found no differences in all three groups over a 24 months follow-up (1). Surgical excision had a significant reduction in the domains of social functioning and role limitations due to physical functioning. These populations cannot be compared directly as the populations were different in both age and tumor size (patients managed by interval scanning were older and had smaller tumors).

\section{DISCUSSION}

Three main policies are now accepted, watchful waiting, surgery and radiotherapy. Each has its indications, its risks and its effect on patients' quality of life. The estimation of possible risks and benefits for the patient is of utmost importance in decision-making (27). The evaluation of quality of life issues can be done using standard forms (SF36, etc.), specific handicap indices (DHI, etc.) or custom questionnaires (PANQOL). Most of these tools, although exceedingly valuable, do not provide a global view of the problem in a patient-oriented perspective taking in consideration the particularities of each therapeutic approach.

The main post-therapeutic sequels include hearing loss, facial nerve dysfunction, balance disorders, pain, other cranial nerve dysfunctions and psychological problems. Any QOL measure should look at both pre-existing handicaps and post-interventional changes in each factor. The pathology is the same but the methods of managements are completely different in both their aim and impact. QOL evaluation forms for vestibular schwannomas should be modified according to the type of management.

It is illogical to list hearing loss as an important issue after translabyrinthine excision in patients who did not have pre-operative serviceable hearing especially that hearing preservation was not associated with any improvement in QOL after surgery. Facial paralysis is a devastating event, which can affect many social and professional functions. Its psychological effect is also significant (21). Patients sched- 
uled for surgery with impaired hearing due to $\mathrm{AN}$ had enough time to adjust their lives for unilateral hearing and thus will not be further affected. On the other hand patients undergoing GK treatment do not expect any hearing deterioration (as widely advertised on the internet or in centers offering this therapy) and thus with any deterioration of hearing they will start complaining albeit after a variable interval.

Acute post-operative gait and balance disturbances are less commonly reported but may affect physical functioning and professional rehabilitation as well as sports and other leisurely activities. Patients may need early gait and balance rehabilitation or a change in vocation (22).

Facial pain and headache have been reported by patients as distressing and may adversely affect their psychological outcome and increase their morbidity and dependence.

Psychological problems such as anxiety, frustration and depression have been reported by patients diagnosed with many diseases. In the case of patients with vestibular schwannomas they are aware that it is a benign disorder that can be effectively controlled. In most studies reporting on this issue, the strongest determinant factor was social support and counseling. Post-treatment resumption of activities and jobs were also associated with a better psychological outcome. However, excessive care may lead to undue dependence and prolonged time out of work. Neuropsychological investigations for cognitive and mnestic performance following procedures in the cerebellopontine angle have highlighted problem areas receiving hitherto little attention (12-14).

Patient selection is not random. Most patients scheduled for a wait and see policy have small tumors, near normal hearing and minimal initial morbidity. Patients selected for GKS have in principle tumors $<3 \mathrm{~cm}$, near normal hearing and may be older. On the other hand patients undergoing microsurgery tend to have larger tumors, poorer initial hearing and possibly other morbidities $(2,23,24)$. Similarly, morbidities may appear over a long period of time so that delayed effects should be taken into consideration and an actuarial model of progressive and delayed morbidity should be applied especially in patients undergoing GK therapy or in a wait and see program. Although surgery is associated with the highest rate of acute morbidity and the highest immediate effect on the QOL of patients, the effects tend to diminish over time whereas in the two other strategies the effects tend to increase. Tumor control with radiotherapy is reasonable $(25,26)$ but there are a number of complications which progress over time, namely: hearing loss, facial paralysis, trigeminal neuropathy, hydrocephalus, vascular injuries and the possibility of malignant transformation (27-32).

Conservative management of vestibular schwannomas carries difficulties: long-term followup of the patients and unpredictability of the tumor growth pattern. A reliable and reproducible radiologic method for evaluating tumor size is of great importance. Sequential MRI cannot be substituted by audiologic examinations solely because increase in VS volume does not correlate with audiologic deterioration significantly (33-35). Wait and see is not associated with any active intervention and follows the natural history of the disease. When the tumor starts to grow and/or cause additional symptoms, only then should the patients be included in the surveys (36). Surgery is often associated with immediate effects but some improve or can be corrected over time. Radiation therapy carries minimal immediate morbidity but possible (so far incompletely clear) long term effects (39). Reporting QOL issues in this context should consider both short and long term effects $(26,36)$.

Thus, patients should be counseled regarding this risk and generic QOL measures such as the SF36 should be used with caution in future assessments (37).

\section{CONCLUSIONS}

When evaluating QOL issues in patients with vestibular schwannoma, the type of intervention as well as the pre-interventional status should be considered (38). The pathology is the same but the methods of managements are completely different in both their aim and impact. Any QOL evaluation in such patients should emphasize the following: hearing, balance and facial paralysis grading and their effect on social and professional performance; general performance as well as the psychological sequels of management.

Standard forms are inadequate to cover the all the functional aspects of the problem. Generic forms 
on the other hand cannot differentiate between the different forms of management. Thus more specific comprehensive systems of evaluation should be devised through the cooperation of multiple centers with different management policies and variable patient populations as well.

\section{REFERENCES}

1. Di Maio S, Akagami R. Prospective comparison of quality of life before and after observation, radiation, or surgery for vestibular schwannomas. J Neurosurg. 2009 Oct;111(4):855-62.

2. Gal TJ, Shinn J, Huang B. Current epidemiology and management trends in acoustic neuroma. Otolaryngol Head Neck Surg. 2010;142, 677-81.

3. Martin HC, Sethi J, Lang D, Neil-Dwyer G, Lutman ME, Yardley L. Patient-assessed outcomes after excision of acoustic neuroma: postoperative symptoms and quality of life. J Neurosurg. 2000;94:211-6.

4. Wu CX. Impact of surgery on health-related quality of life in patients with acoustic neuroma [thesis]. [LA]: University of Southern California; Dec 2006.

5. Tos T, Thomasen PC, Stangerup SE, Tos M, Thomsen J. Patients' fears, expectations and satisfaction in relation to management of vestibular schwannoma: a comparison of surgery and observation. Acta Otolaryngol. 2003;123:600-5.

6. Iyer AK, Gunn R, Sillars H. Quality of Life \& Hearing Preservation in Acoustic Neuroma. Otolaryngology - Head Neck Surg. 2006;135(No 2S):P248.

7. Cross T, Sheard CE, Garrud P, Nikolopoulos TP, O'Donoghue GM. Impact of facial paralysis on patients with acoustic neuroma. Laryngoscope. 2000 Sep;110(9):1539-42.

8. Magliulo G, Zardo F, Damico R, Varacalli S, Forino M. Acoustic neuroma: postoperative quality of life. J Otolaryngol. 2000;29(6):344-7.

9. Saito DM, Cheung SW. A comparison of facial nerve disability between patients with Bell's palsy and vestibular schwannoma. J Clin Neurosc. 2010(17):1122-1125.

10. Myrseth E. Clinical studies of Vestibular schwannomas. [PhD dissertation]. [Bergen]: University of Bergen; 2009.

11. Shaffer BT, Cohen MS, Bigelow DC, Ruckenstein MG. Validation of a disease-specific quality-of-life instrument for acoustic neuroma: the Penn acous- tic neuroma quality-of-life scale. Laryngoscope. 2010;120:1646-54.

12. Bateman $\mathrm{N}$, Nikolopoulos TP, Robinson $\mathrm{K}$, O’Donoghue GM. Impairments, disabilities, and handicaps after acoustic neuroma surgery. Clin Otolaryngol. 1999:25:51-4.

13. Andersson G, Rask-Andersen H. Self-reported hearing problems after surgery for vestibular schwannoma. J Audiol Med. 2002;11(1):25-34.

14. Brooker J, Burney S, Fletcher J, Dally M. A qualitative exploration of quality of life among individuals diagnosed with an acoustic neuroma. Brit J Health Psych. 2009;14:563-78.

15. Lasalletta L, Alfonso C, del Rio L, Roda JM, Gavilan J. (2006) Impact of facial dysfunction on quality of life after vestibular schwannoma surgery. Ann Otol Rhinol Laryngol. 2006;115(9):694-98.

16. Myrseth E, Møller P, Pedersen PH, Lund-Johansen M. Vestibular schwannoma: surgery or gamma knife radiosurgery? A prospective, nonrandomized study. Neurosurgery. 2009 Apr;64(4):654-61.

17. Martin HC, Sethi J, Lang D, Neil-Dwyer G, Lutman ME, Yardley LJ. Patient-assessed outcomes after excision of acoustic neuroma: post-operative symptoms and quality of life. J Neurosurg. 2001;94(2):211-16.

18. Schankin CJ, Gall C, Straube A. Headache syndromes after acoustic neuroma surgery and their Implications for quality of life. Cephalalgia. 2009;29:760-71.

19. Ryzenman JM, Pensak ML, Tew JM Jr. Facial paralysis and surgical rehabilitation: a quality of life analysis in a cohort of 1,595 patients after acoustic neuroma surgery. Otol Neurotol. 2005 May;26(3):516-21.

20. Rameh C, Magnan J. Quality of life of patients following stages III-IV vestibular schwannoma surgery using the retrosigmoid and translabyrinthine approaches. Auris Nasus Larynx. 2010;37:546-552.

21. Pritchard C, Clapham L, Foulkes L, Lang DA, NeilDwyer G. Comparison of cohorts of elective and emergency neurosurgical patients: Psychosocial Outcomes of Acoustic Neuroma and Aneurysmal Sub Arachnoid Hemorrhage Patients and Carers. Surg Neurol. 2004;62:7-16.

22. Parietti-Winkler C, Gauchard A, Simon C, Perrina PP. Pre-operative vestibular pattern and balance compensation after vestibular schwannoma surgery. Neuroscience. 2011;172:285-292. 
23. Dugar J, Nikolopoulos TP, O’donoghue GM. Hearing preservation in acoustic neuroma surgery: the impact of different patient selection criteria. Laryngoscope. 2002;112:2051-53.

24. Mendenhall WM, Friedman WA, Amdur RJ, Antonelli PJ. Management of acoustic schwannoma. Am J Otolaryngol. 2004;25:38-47.

25. Conley G, Hirsch S, Barry E. Stereotactic radiation treatment of vestibular schwannoma: indications, limitations, and outcomes. Curr Opin Otolaryngol Head Neck Surg. 2010 Oct;18(5):351-6.

26. Hsu PW, Chang CN, Lee ST, Huang YC, Chen HC. Outcomes of 75 patients over 12 years treated for acoustic neuromas with linear accelerator-based radiosurgery. J Clin Neurosci. 2010;17:556-60.

27. Arthurs BJ, Lamoreaux WT, Giddings NA, Fairbanks RK, Mackay AR, Demakas JJ, Cooke BS, Lee CM. Gamma knife radiosurgery for vestibular schwannoma: case report and review of the literature. World J Surg Oncol. 2009 Dec 18;7:100.

28. Kim, KN, Park CK, Chung HT, Paek SA, Jung HW, Kim DJ. Long-term outcomes of gamma knife Stereotactic radiosurgery of vestibular schwannomas. J Korean Neurosurg Soc. 2007;42:286-92.

29. Davidson L, Zada G, Yu C, Petrovich Z, Pagnini PG, Zee C, Giannotta SL, Zelman V, Apuzzo MLJ. Delayed toxicity from gamma knife radiosurgery to lesions in and adjacent to the brainstem. J Clin Neurosci. 2009;16:1139-47.

30. Sakamotoa T, Shiratob H, Takeichia N, Aoyamab H, Fukudaa S, Miyasaka K. Annual rate of hearing loss falls after fractionated stereotactic irradiation for vestibular schwannoma. Radioth Oncol. 2001;60:45-48.

31. Kapoor S, Batra S, Carson K, Shuck J, Kharkar S, Gandhi R, Jackson J, Wemmer J, Terezakis S, Shokek O, Kleinberg L, Rigamonti D. Long-term outcomes of vestibular schwannomas treated with fractionated stereotactic radiotherapy: an institutional experience. Int J Radiat Oncol Biol Phys. 2011 Nov 1;81(3):647-53.

32. Mandl ES, Meijer OWM, Slotman BJ, Vandertop WP, Peerdeman SM. Stereotactic radiation therapy for large vestibular schwannomas. Radiotherapy and Oncology. 2010;95:94-98.

33. Shin YJ, Fraysse B, Cognard C, Gafsi I, Charlet JP, Berges C, Deguine O, Tremoulet M. Effectiveness of conservative management of Acoustic neuromas. Am J Otol; 2000;21:857-862.
34. Agrawal Y, Clark JH, Limb CJ, Niparko JK, Francis HW. Predictors of vestibular schwannoma growth and clinical implications., Otol Neurotol. 2010 Jul;31(5):807-12.

35. Shin YJ, Lapeyre-Mestre M, Gafsi I, Cognard C, Deguine O, Tremoulet M, Fraysse B. Neurotological complications after radiosurgery versus conservative management in acoustic neuromas: a systematic review-based study. Acta otolaryngol. 2003;123:59-64.

36. van de Langenberg R, de Bondt BJ, Nelemans PJ, Dohmen AJ, Baumert BG, Stokroos RJ. Predictors of volumetric growth and auditory deterioration in vestibular schwannomas followed in a wait and scan policy. Otol Neurotol. 2011 Feb;32(2):338-44.

37. Fayad J, Semaan M, Lin J, Berliner K, Brackmann D. Conservative management of vestibular schwannoma: expectations based on the length of the observation period. Otol Neurotol. 2014 Aug;35(7):1258-65.

38. Godefroy WP, Kaptein AA, Vogel JJ, van der Mey AG. Conservative treatment of vestibular schwannoma: a follow-up study on clinical and quality-oflife outcome. Otol Neurotol. 2009 Oct;30(7):968-74.

39. Timmer FCA, van Haren AEP, Mulder JJS, Hanssens PEJ, van Overbeeke JJ, Cremers CWRJ, Graamans K. Quality of life after gamma knife radiosurgery treatment in patients with a vestibular schwannoma: the patient's perspective. Eur Arch Otorhinolaryngol. 2010;267:867-73.

40. Sandooram D, Grunfeld EA, Mc Kinney C, Gleeson MJ. Quality of life following microsurgery, radiosurgery and conservative management for unilateral vestibular schwannoma. Clin Otolaryngol. 2004;29:621-7. 